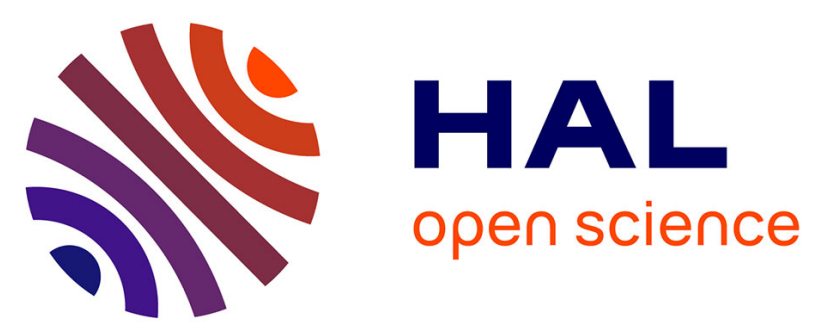

\title{
Frequency of capsid substitutions associated with GS-6207 in vitro resistance in HIV-1 from antiretroviral-naive and -experienced patients
}

Anne-Geneviève Marcelin, Charlotte Charpentier, Aude Jary, Marine Perrier, Nicolas Margot, Christian Callebaut, Vincent Calvez, Diane Descamps

\section{To cite this version:}

Anne-Geneviève Marcelin, Charlotte Charpentier, Aude Jary, Marine Perrier, Nicolas Margot, et al.. Frequency of capsid substitutions associated with GS-6207 in vitro resistance in HIV-1 from antiretroviral-naive and -experienced patients: Resistance to HIV capsid inhibitors. Journal of Antimicrobial Chemotherapy, 2020, 75 (6), pp.1588-1590. 10.1093/jac/dkaa060 . hal-02949451

\section{HAL Id: hal-02949451 \\ https: / hal.sorbonne-universite.fr/hal-02949451}

Submitted on 25 Sep 2020

HAL is a multi-disciplinary open access archive for the deposit and dissemination of scientific research documents, whether they are published or not. The documents may come from teaching and research institutions in France or abroad, or from public or private research centers.
L'archive ouverte pluridisciplinaire HAL, est destinée au dépôt et à la diffusion de documents scientifiques de niveau recherche, publiés ou non, émanant des établissements d'enseignement et de recherche français ou étrangers, des laboratoires publics ou privés. 


\section{Frequency of capsid substitutions associated with GS-6207 in vitro resistance in HIV-1}

\section{from antiretroviral-naïve and -experienced patients}

3

4 Anne-Geneviève MARCELIN ${ }^{1 *}$, Charlotte CHARPENTIER $^{2}$, Aude JARY ${ }^{1}$, Marine 5 PERRIER $^{2}$, Nicolas MARGOT ${ }^{3}$, Christian CALLEBAUT $^{3}$, Vincent CALVEZ ${ }^{1}$, and Diane 6 DESCAMPS $^{2}$

7

$8{ }^{1}$ Sorbonne Université, INSERM, Institut Pierre Louis d'Epidémiologie et de Santé Publique, 9 AP-HP, Hôpitaux Universitaires Pitié-Salpêtrière - Charles Foix, laboratoire de virologie, F75013, Paris, France

${ }^{2}$ Université de Paris, IAME, UMR1137, Inserm, Laboratoire de Virologie, Hôpital BichatClaude Bernard, AP-HP, Paris, France

${ }^{3}$ Gilead Sciences, Foster City, USA

Corresponding author:

Pr. Anne-Geneviève Marcelin

Address: Hôpital Pitié-Salpêtrière, 83 bd de l’hôpital, 75013, Paris, France

Tel: +33142177401

Fax: +33142177411

Email: anne-genevieve.marcelin@aphp.fr

Short running title: Resistance to HIV capsid inhibitors 
Abstract

27 Background: GS-6207 is a first-in-class HIV capsid inhibitor, targeting several functions of HIV capsid in the viral cycle, including viral particles assembly, capsid formation and nuclear entry. GS-6207 has demonstrated picomolar potency in vitro, activity confirmed by high potency in Phase 1 clinical study, with a long acting antiretroviral profile with potential dosing every 6 months. In vitro resistance selections previously conducted with increasing doses of GS-6207 have identified capsid variants with reduced susceptibility to GS-6207.

Objectives: We have studied the prevalence of capsid mutations associated with in vitro resistance to GS-6207 in people living with HIV (PLWH).

Patients and methods: Plasma samples from ART-naïve or ART-experienced PLWH, including PI-experienced, were sequenced, and analyzed for the presence of capsid variants identified during in vitro resistance selections: L56I, M66I, Q67H, K70N, N74D, N74S and T107N.

Results: Among the samples from the 1500 patients studied, none of the seven GS-6207 resistance mutations identified during in vitro selection experiments was detected, regardless of HIV subtype or PLWH treatment history.

Conclusions: Out of the seven HIV capsid substitutions previously selected in vitro and shown to confer phenotypic resistance to GS-6207, none of those seven mutations was observed in this large dataset, suggesting that neither PLWH with previous PI failure nor PLWH with emergence of PI resistance mutations are anticipated to impact GS-6207 activity in these diverse HIV infected populations. 
49 The HIV capsid protein (commonly known as p24) is generated upon cleavage of the HIV gag polyprotein by HIV protease. It provides multiple essential functions throughout the viral

51 replication cycle, making it an attractive target for antiviral intervention. ${ }^{1}$ GS-6207 is a firstin-class HIV capsid inhibitor with a unique, multi-stage mechanism of action, including the inhibition of HIV assembly, proper viral capsid formation, and nuclear entry of viral DNA. In vitro characterization of the HIV capsid inhibitor GS-6207 revealed the high potency of the compound, with a reported $\mathrm{EC}_{50}$ as low as $100 \mathrm{pM}$ with antiviral activity against all major HIV-1 subtypes. ${ }^{2}$ The picomolar potency of GS-6207 along with its unique physicochemical properties make it a prime candidate for a long acting agent. These long acting properties were recently demonstrated in Phase 1a clinical study ${ }^{3}$ and showed that GS-6207 had the potential to be dosed every 6 months. ${ }^{4}$ Subsequently, a Phase $1 \mathrm{~b}$ proof-of-concept clinical study showed that single sub-cutaneous (SC) doses of GS-6207 ranging from 50 to $450 \mathrm{mg}$ resulted in potent antiviral activity in people living with HIV (PLWH), with mean maximum HIV-1 RNA declines ranging from 1.8 to $2.2 \log 10 \mathrm{HIV}$ copies/mL over 10 days. ${ }^{5}$ As a first in class compound, the HIV capsid inhibitor GS-6207 exhibits a unique in vitro resistance profile relative to existing antiretroviral agents. In vitro dose escalation and viral breakthrough selections performed with GS-6207 in T-cell lines and PBMCs infected with clonal and clinical HIV-1 isolates, respectively, identified the HIV CA variants L56I, M66I, Q67H, K70N, N74D, N74S and T107N (alone and in different combinations), with Q67H and N74D being the most predominantly observed variants. ${ }^{6}$ All the GS-6207-selected variants showed reduced susceptibility to GS-6207 and all but Q67H showed reduced infectivity in Tcell lines and impaired replication capacity in primary human CD4+ T-cells. ${ }^{6}$

Genetic variations in gag can naturally occur depending on the HIV subtypes, immune pressure (CTL epitopes), and prior use of HIV protease (PR) inhibitors, as the gag polyprotein 
73 is the substrate for PR. Here, we studied the prevalence of CA mutations ${ }^{6}$ previously 74 identified to be associated with in vitro resistance to GS-6207 in antiretroviral treatment 75 (ART)-naïve or -experienced people living with HIV (PLWH), including people with prior 76 use of PIs.

78 Materials and methods

79 Study population

80 Samples were from patients from the laboratories of Pitié-Salpêtrière and Bichat-Claude 81 Bernard hospitals, Paris, France. Patients were followed according to the local standard of 82 care and resistance testing was performed following the French national recommendations: 83 systematic resistance testing in any new HIV-1 diagnostic and in any ART failure (defined by 84 the occurrence of 2 consecutive plasma HIV- 1 viral load > 50 copies $/ \mathrm{mL}$ ). Plasma samples were from 1500 subjects: ART-Naïve, ART-Experienced with not any PI use in their therapeutic history and ART-Experienced with at least one PI failure episode in their 87 treatment history.

Ethics

The research was conducted in accordance with the Declaration of Helsinki and national and institutional standards. All the patients gave their written informed consent to have their medical chart recorded in the electronic medical record system Nadis® (www.dataids.org; CNIL number: 770134, 30 October 2001).

95 Sequencing

96 Sanger sequencing method was used to sequence HIV gag and protease using as previously 97 described. ${ }^{7-9}$ Sequence information was used to evaluate the presence of mutations 
previously identified in GS-6207 in vitro-selections (L56I, M66I, Q67H, K70N, N74D, N74S, and T107N in CA). ${ }^{6}$ We studied these substitutions in 3 large groups of PLWH: ART-naïve, ART-experienced without PI treatment experience, and ART-experienced with previous PI failure with or without major PI resistance mutations. PI resistance mutations were classified according to IAS-USA list of mutations (www.iasusa.org). ${ }^{10}$

\section{Results}

Among plasma samples of the subjects studied, ART-Naïve $(n=500)$, ART-Experienced without PI use ( $n=500)$, and ART-Experienced with history of PI failure ( $n=500)$, the most prevalent HIV-1 subtypes were B and CRF 02 AG. These results matched the expected subtypes, based of the geographic origin of the PLWH in this analysis. The other subtypes observed were in accordance with the HIV-1 epidemiology in West Africa (Table 1). The RAMs detected in these groups of patients were analyzed for resistance related to PI. In the ART-Naïve group and in the ART-Experienced without PI use group $0.6 \%$ and $0.4 \%$ of major PI RAMs were found respectively, however majority (52.8\%) of ART-Experienced patients with history of PI failure had at least 1 major PI resistance mutation (Table 2). None of the seven GS-6207 resistance mutations identified during in vitro selection experiments (L56I, M66I, Q67H, K70N, N74D, N74S, and T107N) was detected among either the ART-Naïve (500) or the ART-Experienced patients (1000) studied, regardless of HIV-1 subtype or history of PI failure in the therapeutic history. In contrast, maturation inhibitor RAMs in gag ${ }^{11}$ were present, with V362I found in 6.4\% of ART-Naïve, 5.8\% of ART-Experienced without PI use and $9.6 \%$ of ART-Experienced with history of PI failure, and V370A found in $9.8 \%$ of ARTNaïve, $8.4 \%$ of ART-Experienced without PI use and 17\% of ART-Experienced with history of PI failure. Similarly, major PI-RAMs (Table 2) were present in the PI-experienced group 
122 (52.8\%). Overall, the highly conserved region of HIV capsid identified as the binding site for

123 GS-6207 displayed very low variability.

124 Discussion

125 The genotypic analyses conducted here are contributing to document the absence of naturally 126 occurring resistance mutations against GS-6207 in different populations of PLWH. The 127 subtype distribution captured by this large number of samples analyzed covered a wide range 128 of HIV geographical distribution, representative of many subtypes circulating throughout the world. This suggests that GS-6207 has the potential to be active in vivo regardless of HIV-1 subtypes, including complex recombination forms. This observation is similar to previous

131 observations made by Perrier et al. ${ }^{7}$ for a related inhibitor of the CAI class, GS-CA-1. ${ }^{12}$

132 However, the novelty of this study was genotyping of patients who were ART-experienced 133 with the PI-experienced subset showing a high frequency (52.8\%) of acquired PI resistance mutations/polymorphisms. Furthermore, the analysis of these 3 cohorts, of ART-naïve and experienced PLWH ( $\mathrm{n}=1500$ overall), also showed that previous PI failure and emergence of PI resistance mutations did not lead to the presence of potential resistance to GS-6207 in 137 HIV-1 CA. Although the lack of pre-existing resistance mutations could be anticipated for a 138 first in class such as GS-6207, these results suggest that the HIV CA inhibitor GS-6207 has a 139 very low likelihood of pre-existing resistance mutations in the PLWH population. As a first140 in-class compound, the resistance profile GS-6207 has yet to be fully established. Limitations 141 of our study include the fact that the reported in vitro drug selections with GS-6207 were only 142 performed for 100 days. Given the potency of capsid inhibitors, resistance may require more 143 extended time to develop. Additionally, the use of ultra-deep sequencing. could have 144 characterize these mutations even further. Overall, these data showing the absence of natural 145 variability at potential RAM positions, suggests that GS-6207 has the potential to be effective 146 regardless of treatment history or prior PI use. 


\section{Acknowledgements}

This work has been previously presented at 17th European AIDS Conference - Nov 6-9, 2019

- Basel, Switzerland (PE13/15 Poster).

Funding

154 This work has been funded by Agence Nationale de Recherches sur le SIDA et les hépatites 155 virales, and by Gilead Sciences.

\section{Transparency declarations}

158 AGM, CC, DD and VC have received grants and honoraria from Janssen-Cilag, Gilead, MSD and VIIV Healthcare. AJ and MP have none to declare.

\section{References}

162 1. Singh K, Gallazzi F, Hill KJ, et al. GS-CA Compounds: First-In-Class HIV-1 Capsid Inhibitors 163 Covering Multiple Grounds. Front Microbiol 2019; 10: 1227.

164 2. Yant SR, Mulato A, Stepan GJ, et al. GS-6207, a potent and selective first-in-class long165 acting hiv-1 capsid inhibitor - Conference on Retroviruses and Opportunistic Infections, 166 Seattle, WA, March 4-7, 2019 - poster 1504.

167 3. Sager JE, Begley R, Rhee MS, et al. Safety and PK of Subcutaneous GS-6207, a Novel HIV-1 168 Capsid Inhibitor - Conference on Retroviruses and Opportunistic Infections 2019, Seattle, 169 WA, 4-7 March 2019 - oral presentation 0-13.

170 4. Sager JE, Begley R, Rhee MS, et al. Safety and PK of subcutaneous GS-6207, a novel HIV-1 171 capsid inhibitor - European AIDS Clinical Society 2019, Basel, Switzerland - oral presentation 172 PS13/1.

173 5. Daar ES, McDonald C, Crofoot G, et al. Safety and Antiviral Activity Over 10 Days Following 174 a Single Dose of Subcutaneous GS-6207, a First-in-Class, Long-Acting HIV Capsid Inhibitor in 175 People Living With HIV - 10th IAS Conference on HIV Science, 21-24 July 2019, Mexico City, 176 Mexico - oral presentation LBPE3/17. 
177 6. Yant SR, Mulato A, Hansen D, et al. In Vitro Resistance Profile of GS-6207, a First-in-Class 178 Picomolar HIV Capsid Inhibitor in Clinical Development as a Novel Long-Acting Antiretroviral 179 Agent - 10th IAS Conference on HIV Science, 21-24 July 2019, Mexico City, Mexico - poster 180 TUPEA075.

181 7. Perrier $M$, Bertine $M$, Le Hingrat $Q$, et al. Prevalence of gag mutations associated with in 182 vitro resistance to capsid inhibitor GS-CA1 in HIV-1 antiretroviral-naive patients. J Antimicrob 183 Chemother 2017; 72: 2954-5.

184 8. Castain L, Perrier M, Charpentier C, et al. New mechanisms of resistance in virological 185 failure to protease inhibitors: selection of non-described protease, Gag and Gp41 mutations. 186 J Antimicrob Chemother 2019; 74: 2019-23.

187 9. Lambert-Niclot S, Flandre $\mathrm{P}$, Malet I, et al. Impact of gag mutations on selection of 188 darunavir resistance mutations in HIV-1 protease. J Antimicrob Chemother 2008; 62: 905-8.

189 10. Wensing AM, Calvez V, Ceccherini-Silberstein F, et al. 2019 update of the drug resistance 190 mutations in HIV-1. Top Antivir Med 2019; 27: 111-21.

191 11. Margot NA, Gibbs CS, Miller MD. Phenotypic susceptibility to bevirimat in isolates from 192 HIV-1-infected patients without prior exposure to bevirimat. Antimicrob Agents Chemother 193 2010; 54: 2345-53.

194 12. Yant SR, Mulato A, Hansen D, et al. A highly potent long-acting small-molecule HIV-1 195 capsid inhibitor with efficacy in a humanized mouse model. Nat Med 2019; 25: 1377-84. 
197 Table 1. Distribution of HIV-1 subtypes among studied patients

\begin{tabular}{lccc}
\hline HIV-1 Subtype & ARV-Naïve & ARV-Experienced & ARV-Experienced \\
Distribution, \% & $(\mathbf{n = 5 0 0 )}$ & no PI use & PI failure history \\
(n) & & $(\mathbf{n = 5 0 0 )}$ & $(\mathbf{n = 5 0 0 )}$ \\
\hline B & $37 \%(185)$ & $42 \%(210)$ & $56 \%(280)$ \\
CRF02_AG & $46 \%(230)$ & $48 \%(240)$ & $37 \%(185)$ \\
F1 & $4.6 \%(23)$ & $2.4 \%(12)$ & - \\
CRF06 & $4.4 \%(22)$ & $3.8 \%(19)$ & $3.4 \%(17)$ \\
A1 & $2.8 \%(14)$ & - & - \\
D & $2.2 \%(11)$ & $2.2 \%(11)$ & $1.6 \%(8)$ \\
Other non-B & $3.0 \%(15)$ & $1.6 \%(8)$ & $1.0 \%(5)$
\end{tabular}

198

ARV, antiretroviral; PI, protease inhibitor

199 

mutations

\begin{tabular}{lccc}
\hline Resistance & $\begin{array}{c}\text { ARV-Naïve } \\
\text { Mutations }\end{array}$ & $\begin{array}{c}\text { ARV-Experienced } \\
\text { no PI use }\end{array}$ & $\begin{array}{c}\text { ARV-Experienced } \\
\text { PI failure history } \\
(\mathbf{n = 5 0 0})\end{array}$ \\
\hline $\begin{array}{l}\text { Number of Major PI } \\
\text { Resistance }\end{array}$ & & & \\
Mutations, \% (n) & & & \\
0 & $99.4 \%(497)$ & $99.6 \%(498)$ & $47.2 \%(236)$ \\
1 & $0.6 \%(3)$ & $0.4 \%(2)$ & $22.4 \%(112)$ \\
2 & - & - & $16.4 \%(82)$ \\
3 & - & - & $10.4 \%(52)$ \\
4 & - & - & $3.6 \%(18)$ \\
\hline
\end{tabular}

GS-6207 Resistance

Mutations (in vitro

selected)

L56I

M66I

Q67H

K70N

N74D

N74S

$\mathrm{T} 107 \mathrm{~N}$

202 PI, protease inhibitor; ARV, antiretroviral; List of major PI resistance mutations according to IAS-USA list of 203 mutations: D30N, V32I, M46I/L, I47A/V, G48V, I50L/V, I54L/M/V, Q58E, T74P, L76V, V82A/F/L/T/S, 204 N83D, I84V, N88S, L90M. 